\title{
Measurement of Residual Urine Volume by Means of Ultrasonic Scanning: A Comparative Study
}

\author{
J. Kjeldsen-Kragh MD \\ Department of Rheumatology, Viborg Hospital, Denmark.
}

\section{Summary}

The basis for this paper was 107 ultrasonic examinations of the bladder in 20 patients with neurogenic bladder dysfunction. The residual urine volume was estimated in three different ways.

1. The residual urine volume is approximately equal to the product of the width, height and depth of the bladder multiplied by a correction factor.

2. By a new method: The residual volume is approximately equal to the crosssectional area of the bladder when measured in the sagittal plane multiplied by the width of the bladder.

3. By the nomogram method: The cross-sectional area in the sagittal and the transverse plane are respectively plotted into a specially designed nomogram giving an estimate of the residual urine volume.

The bladder was emptied by catheterisation immediately after the ultrasonic examinations. Of the three methods, number 2 was found to give the most accurate estimate of residual urine volume.

Key words: Bladder volume; Ultrasonic scanning; Residual urine.

Patients with neurogenic bladder dysfunction often show incomplete emptying of the bladder. During bladder training it is important to make frequent measurements of residual urine volume. In recent years it has been common to do this by ultrasonic scanning.

Many centres carry out ultrasonic measurement of width, height and depth of the bladder. For an estimate of residual urine volume the product of the three dimensions is multiplied by a correction factor. McLean and Edell (1978) and Kornhuber et al. (1980) have further suggested subtraction respectively addition of a certain volume to get the most exact estimate of the residual urine volume. The numerical value of the correction factor varies between 0.46 and 0.79 respectively (McLean and Edell, 1978; Pedersen, 1979; Brunn and Ruf, 1980; Kornhuber et al., 1980). This suggests the problems of applying this form 
of estimation. Furthermore, this method is rather laborious and has a deviation of $21-26 \%$ from the true bladder volume (Poston et al., 1983; Hakenberg et al., 1983).

\section{Methods}

One hundred and seven ultrasonic examinations have been carried out on 20 patients with neurogenic bladder dysfunction applying a real-time B-mode linear scanner with a built-in programme for measuring the cross-sectional area. The transducer worked at a frequency of $3 \mathrm{MHz}$. The weight of the scanner was $10 \mathrm{~kg}$ and it was carried around on a mobile cart. All examinations were carried out bedside with the patients supine.

At each examination the bladder was scanned in the sagittal and in the transverse plane (Figs. 1 and 2). The transducer was positioned in the midline above the symphysis pubis, thus obtaining a sagittal section of the lower abdomen. When the largest picture of the bladder appeared on the monitor the picture was frozen and the bladder's height (h), depth (d) and cross-sectional area (AS) were measured. The transducer was then positioned transversely just above the symphysis pubis, at right angles to the sagittal plane providing another section of the lower abdomen. When the largest picture appeared on the monitor it was frozen and the bladder's width (w), depth (d) and cross-sectional area (AT) were measured.

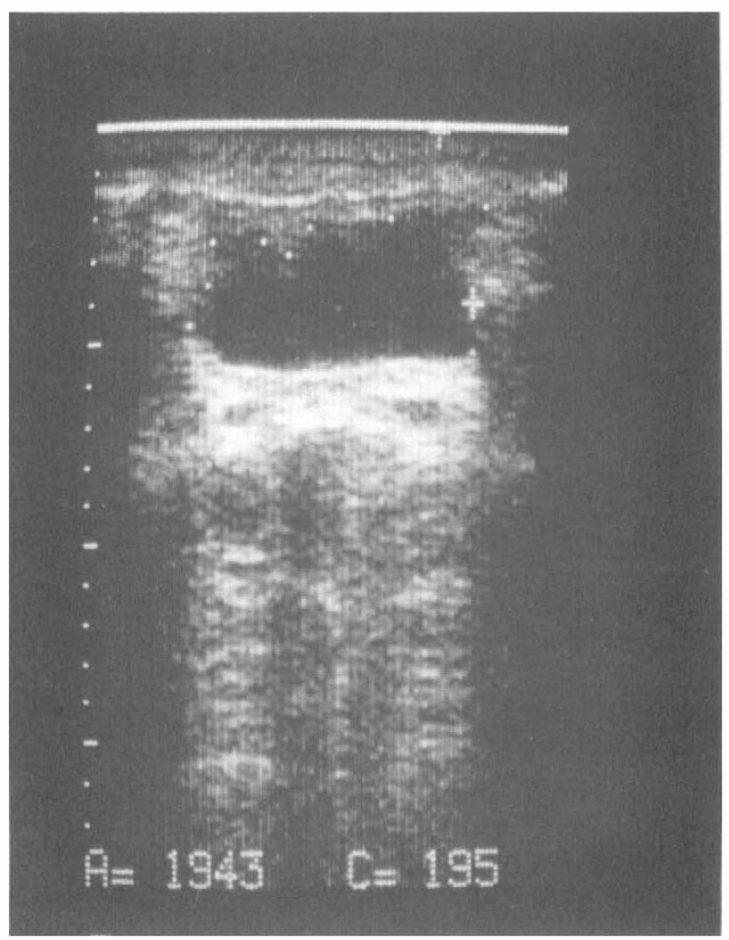

Figure 1 Ultrasonic scan: Transverse scanning. 


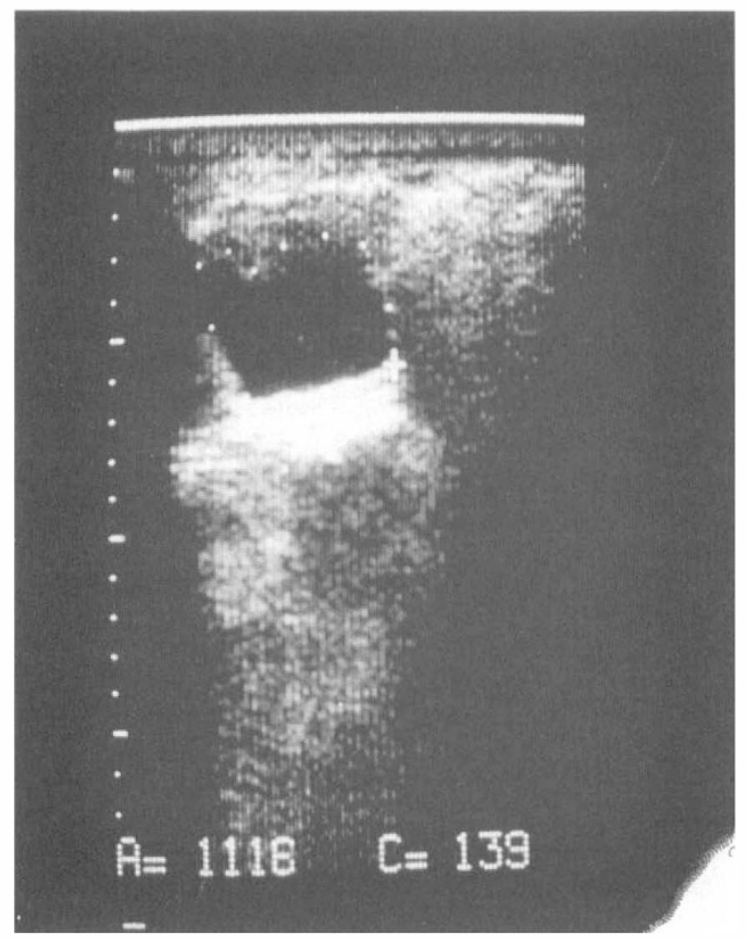

Figure 2 Ultrasonic scan: Sagittal scanning.

Immediately after each ultrasonic examination, the true volume of the bladder $\left(\mathrm{V}_{\mathrm{t}}\right)$, was measured by emptying the bladder by catheterisation. To be quite sure that the bladder was completely emptied manual pressure was applied to the lower abdomen just above the symphysis pubis.

The bladder was emptied within 5 minutes after the scanning in 82 examinations and in the remainder within 10 minutes.

Sometimes there are some difficulties in outlining the contours of the bladder, but in this study no examinations were excluded from the statistical calculations due to such problems.

For each examination h.w.d. and AS.w have been calculated and plotted against $\mathrm{V}_{\mathrm{t}}$. Applying the nomogram method the volume of the bladder $\left(\mathrm{V}_{\text {nom }}\right)$ has also been estimated from AS and AT and plotted against $V_{t}$ (Figs 3-5).

In seven examinations it was impossible to use the nomogram for estimation of residual urine volume because the cross-sectional area in either of the planes was either too great or too small.

\section{Results}

For each of the three methods the correlation between the calculated values and the $\mathrm{V}_{\mathrm{t}}$-values is examined. From Figures 3 and 4 it appears that the deviation of the calculated values from the true volume increases with an increase in $\mathrm{V}_{\mathrm{t}}$ values. For this reason no linear regression analysis has been made on the raw 


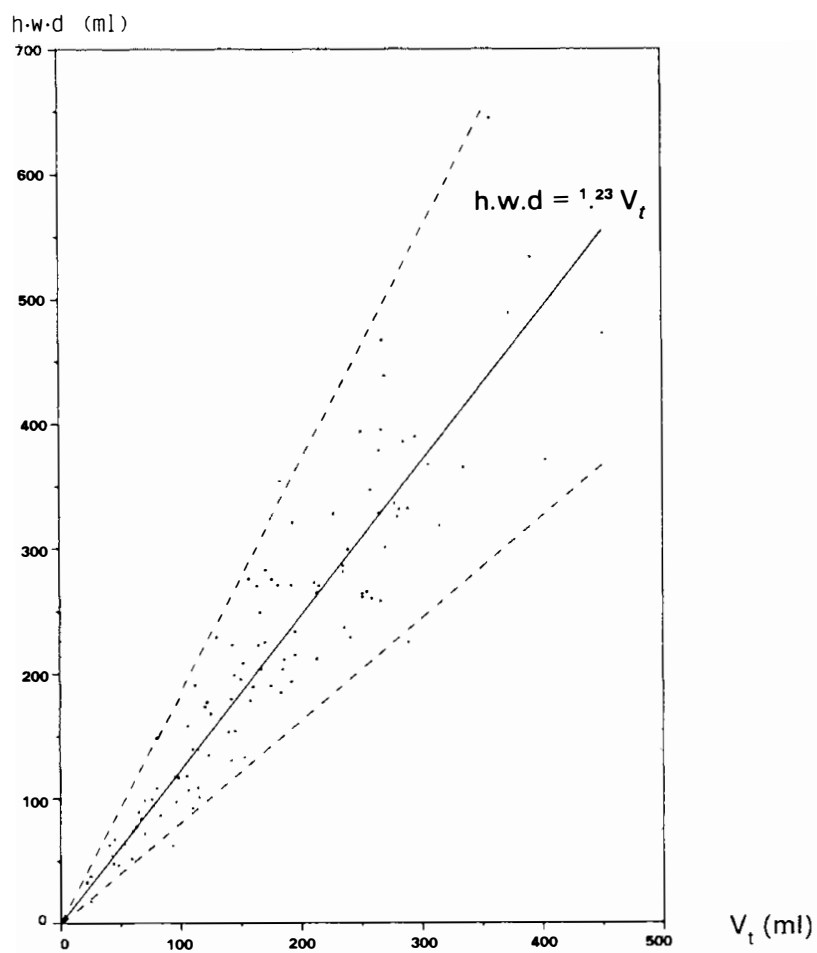

Figure 3 The broken lines correspond approximately to the $95 \%$ confidence limits.

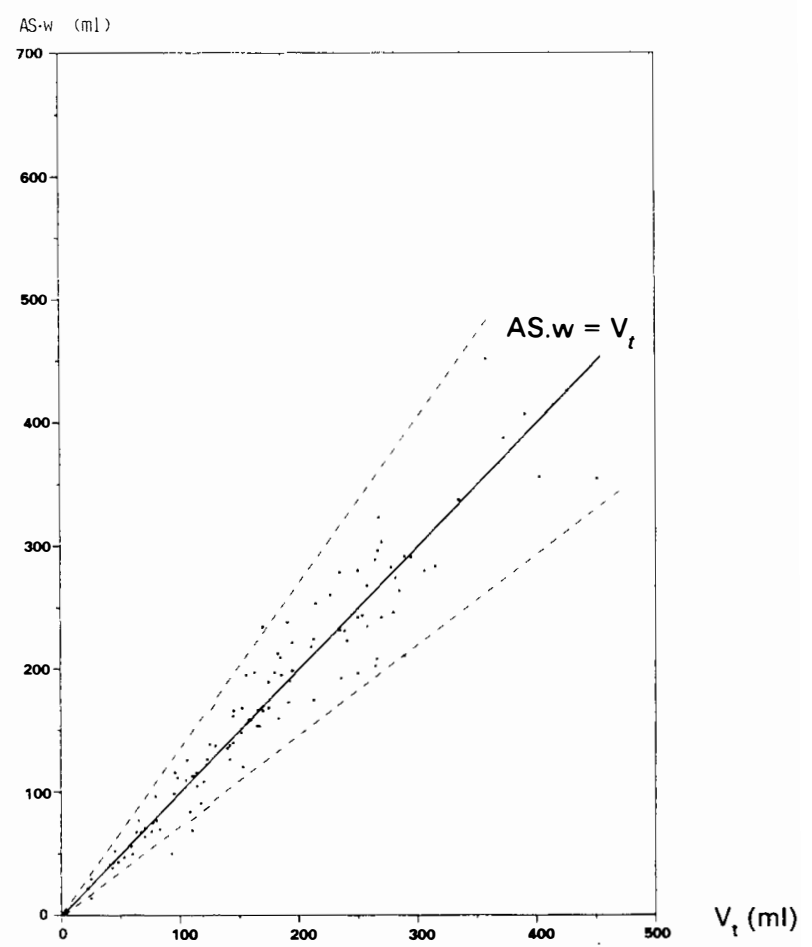

Figure 4 The broken lines correspond approximately to the $95 \%$ confidence limits. 


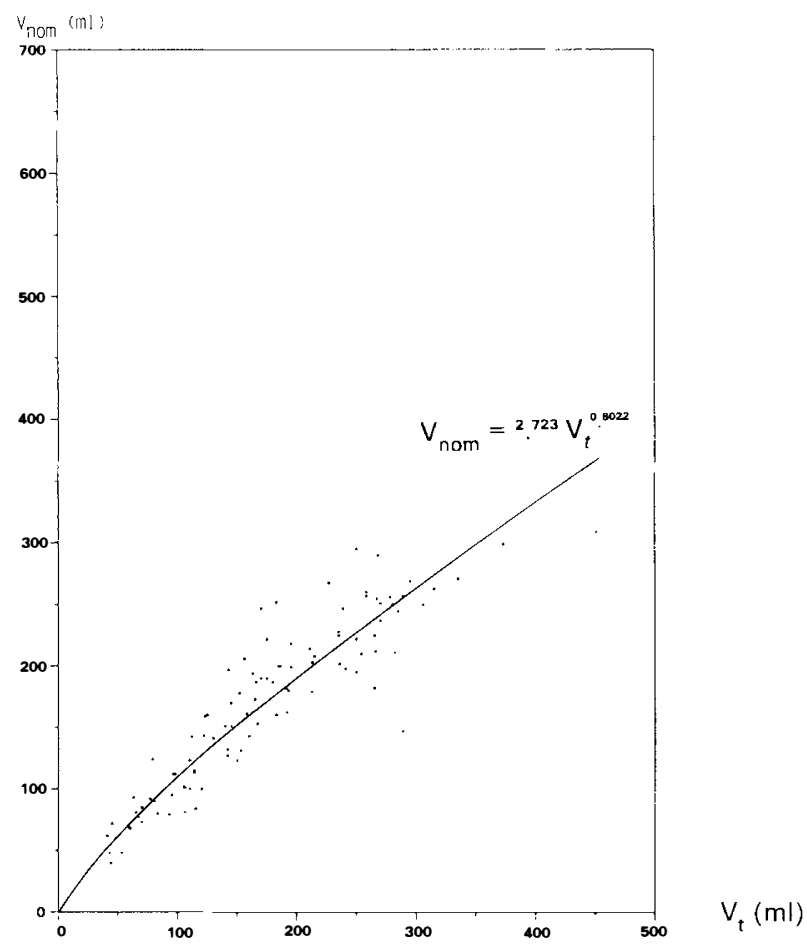

Figure 5 Residual urine volume estimated by the nomogram method (Rageth and Langer, 1982).

numerical material but on the logarithms of the numerical values. The assumption has been made that the curve which describes the relationship between $\mathrm{V}_{\mathrm{t}}$-values and the calculated values begins at the origin.

For each of the three methods, linear regression analysis has been made on the logarithms of the numerical values, and the equation for the regression line, the standard deviation about the regression line (SD) and the correlationcoefficient $(r)$ have been calculated:

1: $\ln (\mathrm{h} . \mathrm{w} . \mathrm{d})=0 \cdot 166+1 \cdot 007 \cdot \ln \mathrm{V}_{\mathrm{t}}$, thus

h.w.d $=1 \cdot 18 \cdot \mathrm{V}_{\mathrm{t}}^{1 \cdot 007}$

$\mathrm{SD}_{\ln \text { (h.w.d) }}=0.209 \quad \gamma_{\ln \text { (h.w.d.) }}=0.95$

For $\mathrm{V}_{\mathrm{t}}$-values from 0 to $500 \mathrm{ml}$

$\mathrm{h} . \mathrm{w} . \mathrm{d} \doteqdot 1 \cdot 23 . \mathrm{V}_{\mathrm{t}}$, or

$\mathrm{V}_{\mathrm{t}} \doteqdot \mathrm{h}$.w.d.0.81

2: $\ln$ (AS.w) $=-0.111+1 \cdot 019 \cdot \ln \mathrm{V}_{\mathrm{t}}$, thus

AS. $w=0 \cdot 90 . \mathrm{V}_{\mathrm{t}}^{1 \cdot 019}$

$\mathrm{SD}_{\ln (\text { AS.w })}=0.156 \quad \gamma_{\ln (\text { AS. } w)}=0.97$

For $\mathrm{V}_{\mathrm{t}}$-values from 0 to $500 \mathrm{ml}$

$\mathrm{V}_{\mathrm{t}} \doteqdot \mathrm{AS}$.w

3: $\ln \mathrm{V}_{\text {nom }}=1 \cdot 002+0 \cdot 8022 \cdot \ln \mathrm{V}_{\mathrm{t}}$, thus

$\mathrm{V}_{\text {nom }}=2 \cdot 723 . \mathrm{V}_{\mathrm{t}}^{0.8022}$

$\mathrm{SD}_{\text {lnV }_{\text {nom }}}=0.163 \quad \gamma_{\text {lnV }_{\text {nom }}}=0.93$ 
The slope of the regression line is significantly different from $1(\mathrm{p}<0.001)$. If one discards the assumption that the line describing the relationship between $\mathrm{V}_{\text {nom }}$ and $\mathrm{V}_{\mathrm{t}}$ starts at the origin and makes linear regression analysis on the raw values of $V_{\text {nom }}$ and $V_{t}$, one gets a regression line with this equation:

$\mathrm{V}_{\text {nom }}=40 \cdot 7+0 \cdot 739 . \mathrm{V}_{\mathrm{t}}$.

Methods 1 and 2 are compared, and the ratio between the variance estimates is F-distributed:

$$
\mathrm{F}=\frac{\left(\mathrm{SD}_{\ln (\mathrm{h} . \mathrm{w} . \mathrm{d})}\right)^{2}}{\left(\mathrm{SD}_{\ln (\mathrm{AS} . \mathrm{w})}\right)^{2}}=\frac{0 \cdot 209^{2}}{0 \cdot 156^{2}}=1 \cdot 80
$$

For 105 degrees of freedom for both variances according to the F-distribution the p-value is: $\mathrm{p}<0.005$.

In every ultrasonic examination the deviation of the estimated residual urine volume from the true volume has been calculated. For method 1, 2 and 3 the average numerical deviation is $28 \%, 11^{\%}$ and $16^{\circ} \%$ respectively.

\section{Discussion}

For method 3 the slope of the regression line is significantly different from 1 , indicating that the $\mathrm{V}_{\text {nom }}-\mathrm{V}_{\mathrm{t}}$-graph is curved. This is rather surprising. One would expect a straight line starting at the origin and with a slope equal to 1 . The extreme position of the two values $(289,147)$ and $(451,309)$ could be suspected for causing the curvature, but even if the two outlying ones were excluded, the slope of the regression line was significantly different from 1 $(\mathrm{p}<0.001)$.

If one makes linear regression analysis on the raw values of $V_{\text {nom }}$ and $V_{t}$, the $\mathrm{V}_{\text {nom }}-\mathrm{V}_{\mathrm{t}}$-graph becomes straight. Unfortunately this straignt line has an intercept with the ordinate of $40.7 \mathrm{ml}$ and a slope of 0.739 making this line inappropriate for estimating the residual urine volume.

Method $1\left(\mathrm{~V}_{\mathrm{t}}=\mathrm{h} . \mathrm{w} \cdot \mathrm{d} .0 \cdot 81\right)$ could possibly be improved by taking into account the bladder's depth transversally scanned. It is quite common that the measurement of the bladder's depth varies a little between scanning in the sagittal and the transverse planes, even though these two measurements are expected to be equal. The correlation between $\mathrm{V}_{\mathrm{t}}$ and $\mathrm{h} \cdot \mathrm{w} \cdot 0 \cdot 5(\mathrm{~d}+\mathrm{D})$ has been examined and the equation for the regression line calculated: $V_{t}=$ h.w.0 5. $(\mathrm{d}+\mathrm{D}) \cdot 0 \cdot 82$. This method seems to be more accurate than method 1 although the difference is not statistically significant.

Method 1 and 2 are compared and method $2\left(V_{t}=\right.$ AS .w) is found to be the most accurate with an average deviation of only $11 \%$, which is considerably better than method $1(28 \%)$ and also in comparison with the results published earlier (Poston et al., 1983; Hakenberg et al., 1983; Rageth and Langer, 1982). The difference between method 1 and 2 is highly significant $(\mathrm{p}<0.005)$.

When methods 2 and 3 are compared there are at least four important points to note: Firstly, the $V_{\text {nom }}-V_{t}$-graph is significantly curved. Because of the curvature there is a tendency by method 3 to overestimate bladder volumes below $158 \mathrm{ml}$ and underestimate volumes above. Secondly, the average numeri- 
cal deviation of the estimated volume from the true volume is $11 \%$ for method 2 and $16 \%$ for method 3 . Thirdly, seven estimations were impossible to do by method 3 because the cross-sectional area in either of the planes was either too great or too small. Fourthly, method 2 is less time consuming than the other two methods. When the scanning procedure is restricted to measure AS and w the whole examination takes less than 3 minutes including the time for multiplying AS and w. For these reasons method 2 is considered to be the best way to estimate the residual urine volume.

A precondition for this study was that the volume by catheterisation really reflected the actual residual urine volume. Because of the problems of emptying the bladder by catheter in supine patients a firm manual suprapubic pressure was applied after each catheterisation, and the pressure was maintained until the urine flow had stopped. Of course in some cases there might have been a small volume which was not emptied by catheterisation. On the other hand, the small volume produced by the kidneys in the period from the ultrasonic scanning was finished until the catheterisation was completed has falsely increased the residual urine volume. When all three methods of estimating residual urine volume were done from the same examination deviations of the $\mathrm{V}_{\mathrm{t}}$-values had the same consequences for all three methods. If this deviation is of the same range for all $\mathrm{V}_{\mathrm{t}}$-values it will not affect the general conclusion about the accuracy of the three methods.

The scanner used in this study has a linear transducer. During scanning in the sagittal plane it was, in many cases, necessary to press the cranial part of the transducer quite deeply into the abdomen in order to see the lowest area of the bladder on the monitor screen. This was especially necessary in cases where the abdomen was either adipose or distended in order to avoid shadowing from the symphysis pubis. It is, therefore, likely that scanning would be easier using a sector-scanner where the shadow-effect from the symfysis pubis is avoided.

If one does not have the scanning equipment with the feature for measuring the cross-sectional area this study shows that method 1 is fairly good in estimating the residual urine volume. Concerning the value of the correction factor for h.w.d, this study suggests 0.81 to be most appropriate, and this is of the same size as found by Kornhuber et al., (1980). Others do not agree about this value of the correction factor (McLean and Edell, 1978; Pedersen, 1979; Brunn and Ruf, 1980). The reason may partly be due to the different equipment used at the different centres. The picture of the bladder varies slightly for the different scanners. The different sections of the contours of the bladder vary in sharpness, and the examiners who use the scanner daily develop a certain routine for demarcating the bladder wall. Given personal variations and different scanning equipment, one would generally expect interobserver variation to a certain extent. How much this is due to systematic errors can be subject for another study.

\section{Acknowledgments}

This study has been supported economically by Rosa og Asta Jensens fond, Viborg sygehus.

I am indebted to Sten Kremmer who has helped me by constructing programs for the statistical calculations and to Wendy Mogensen, who has advised me on language problems. 


\section{References}

BRUNN J, RUF G 1980 Sonographische Zystrometrie. Deutsche Medizinische Wochenschrift 105: 1501-1503.

HAKENBERg OW, Ryall RL, LANGlois SL, MARShall VR 1983 The estimation of bladder volume by sonocystography. Fournal of Urology 130: 249-251.

KoRnhuber HH, WIDDER B, CHRIST K 1980 The Measurement of Residual Urine by Means of Ultrasound (Sonocystography) in Neurogenic Bladder Disturbances. Archiv für Psychiatrie und Nervenkrankheiten 228: 1-6.

MCLEAN GK, EDELL SL 1978 Determination of Bladder Volumes by Gray Scale Ultrasonography. Radiology 128: 181-182.

PEDERSEN JF 1979 Determination of residual urine by ultrasonic scanning (In Danish). Ugeskrift for Laeger 141: 649-650.

Poston GJ, Joseph AEA, Riddle PR 1983 The Accuracy of Ultrasound in the Measurement of Changes in Bladder Volume. British fournal of Urology 55: 361-363.

RAGETH JC, LANGER K 1982 Ultrasonic Assessment of Residual Urine Volume. Urological Research 10: 57-60. 